\title{
Análise de um radioimunoensaio iodado para determinação de 11-deoxicortisol
}

\author{
Analysis of an iodide radioimmunoassay for \\ 11-deoxicortisol measurement
}

João Luiz de Oliveira Madeira', Luciane Zgoda Bussmann', Helena Panteliou Lima-Valassi', Berenice Bilharinho de Mendonça'

\begin{abstract}
'Hospital das Clínicas, Faculdade de Medicina da Universidade de São Paulo (HCFMUSP), Departamento de Clínica Médica, Disciplina de Endocrinologia e Metabologia, Laboratório de Hormônios e Genética Molecular (LIM/42), São Paulo, SP, Brasil
\end{abstract}

Correspondência para: João Luiz de Oliveira Madeira Universidade de São Paulo, Faculdade de Medicina, Universidade de São Paulo PAMB, Laboratório de Hormônios e Genética Molecular LIM/42, Av. Dr. Enéias de Carvalho Aguiar, $155,2^{\circ}$ andar, bloco 6 05403-900 - São Paulo, SP, Brasil joao.madeira@usp.br

Recebido em 25/06/2013 Aceito em 15/01/2014

\section{RESUMO}

Objetivo: Nosso objetivo foi comparar duas técnicas de dosagem do 11-desoxicortisol: a técnica de radioimunoensaio iodado, a qual foi validada neste trabalho, e a cromatografia líquida de alta performance seguida por espectrometria de massa em tandem (LC-MS/MS), sendo a última considerada o padrão-ouro para dosagem dos hormônios esteroides. Materiais e métodos: Para a comparação entre os resultados de 11-desoxicortisol, foram selecionadas 88 amostras. Resultados: A sensibilidade analítica do radioimunoensaio foi de $0,30 \mathrm{ng} / \mathrm{mL}$, com linearidade e perfil de precisão inadequado ( $34 \%$ das amostras com CV $\geq 20 \%$ ). Das 88 amostras selecionadas, apenas 54 apresentaram resultados mensuráveis em ambos os métodos. A comparação desses resultados, por meio da regressão de Deming, resultou em um coeficiente de correlação de 0,610 , inclinação de 3,751, intercepção de 0,145, evidenciando a pobre correlação entre os resultados e a superestimação dos resultados pelo RIA. Conclusão: Concluímos que o método de dosagem de 11-desoxicortisol por radioimunoensaio iodado apresentou resultados inadequados nos diversos parâmetros avaliados, inviabilizando sua utilização como método de dosagem do 11-desoxicortisol. Arq Bras Endocrinol Metab. 2014;58(3):232-6

\section{Descritores}

11-desoxicortisol; radioimunoensaio; espectrometria de massas; hiperplasia suprarrenal congênita

\begin{abstract}
Objective: Our aim was to correlate 11-deoxycortisol levels obtained by two currently available techniques for 11-deoxycortisol measurement: radioimmunoassay, and high performance liquid chromatography followed by tandem mass spectrometry (MS/MS). The latter is the gold standard method for steroid hormone measurement. Materials and methods: We selected 88 samples and the results of these two methods were compared by Deming regression. Results: The analytical sensitivity of the RIA was $0.30 \mathrm{ng} / \mathrm{mL}$, with inadequate linearity and inadequate precision profile ( $34 \%$ of the samples had a CV $\geq 20 \%$ ). From the selected samples, 54 had measurable levels of 11-deoxycortisol in both methods and were used in the comparison. The comparison of RIA with LC-MS/MS showed an overestimation of the results by RIA. The correlation coefficient was 0.610 ; linear regression slope was 3.751 ; and the intercept was 0.145 , indicating a poor correlation between the two methods. Conclusion: We concluded that 11-deoxycortisol measured by radioimmunoassay, despite a good analytical sensitivity, showed very low specificity, precluding its use as a reliable method for 11-deoxycortisol measurement. Arq Bras Endocrinol Metab. 2014;58(3):232-6
\end{abstract}

Keywords

11-deoxycortisol; radioimmunoassay; mass spectrometry; congenital adrenal hyperplasia 


\section{INTRODUÇÃO}

$\mathrm{A}$ hiperplasia adrenal congênita por deficiência de $11 \beta$-hidroxilase (HAC-110HD) é uma doença autossômica recessiva descrita pela primeira vez em 1955 por Eberlein e Bongiovani $(1,2)$ e compreende cerca de $5 \%$ a $8 \%$ dos casos de HAC, com uma incidência de aproximadamente um entre 100.000 nascidos vivos $(3,4)$.

O principal diagnóstico diferencial da HAC-110HD é com a forma clássica virilizante da deficiência de $21 \alpha$-hidroxilase (HAC-21OHD). O diagnóstico da HAC-11OHD deve ser realizado por meio da dosagem de 11-desoxicortisol $(3,4)$, a qual é pouco disponível e apresenta alto custo, de modo que os níveis desse hormônio frequentemente não são mensurados em pacientes com ambiguidade genital. Pacientes com HAC-11OHD geralmente apresentam níveis elevados de 17OHP, acarretando diagnóstico equivocado de HAC-21OHD (3).

A determinação do 11-desoxicortisol foi realizada por vários anos pela técnica de radioimunoensaio triciado após extração do soro com hexano e acetato de etila. Com as restrições para uso de compostos triciados para preservação do meio ambiente, o Laboratório de Hormônios e de Genética Molecular (LIM/42) do Hospital das Clínicas da Faculdade de Medicina da Universidade de São Paulo (HCFMUSP) passou a terceirizar a determinação de 11-desoxicortisol, em um laboratório privado (Laboratório Fleury) que utiliza a técnica de cromatografia líquida de alta performance seguida por espectrometria de massa em tandem (MS/MS) (5).

Recentemente, o kit 11-Desoxycortisol - RIA CT da DiaSource, Nivelles, Bélgica, um radioimunoensaio iodado, foi disponibilizado no mercado nacional. Entretanto, não há dados na literatura da comparação entre esses métodos para dosagem do 11-desoxicortisol.

O diagnóstico preciso da HAC-110HD antes da realização do tratamento é fundamental para reposição hormonal adequada, além de ser importante para dirigir o estudo molecular para posterior aconselhamento genético e tratamento pré-natal.

Nosso objetivo foi verificar o desempenho analítico de um kit comercial para determinação de 11-desoxicortisol e comparar seus resultados com os obtidos pela técnica de LC-MS/MS, considerada padrão-ouro para dosagem dos hormônios esteroides.

\section{MATERIAIS E MÉTODOS}

\section{Validação do radioimunoensaio iodado}

Radioimunoensaio iodado (RIE): foi utilizado o kit 11-Desoxycortisol - RIA - CT (11-DOC, RIA CT, n. cat. KIPI20000, DiaSource, Nivelles, Bélgica) sem extração prévia, conforme as instruções do fabricante. Na validação do RIE, foram analisados os seguintes parâmetros: sensibilidade analítica, exatidão, linearidade, precisão intraensaio, perfil de precisão e especificidade.

Sensibilidade analítica: 10 replicatas do calibrador zero foram dosadas em um único ensaio e o valor da sensibilidade analítica foi definido como a concentração correspondente à média das contagens das replicatas de calibrador zero subtraída de dois desvios-padrão dessas contagens.

Exatidão: foram preparados os padrões de 11-desoxicortisol nas seguintes concentrações: $62,5 \mathrm{ng} / \mathrm{mL}$, $31,25 \mathrm{ng} / \mathrm{mL}, 15,62 \mathrm{ng} / \mathrm{mL}, 7,81 \mathrm{ng} / \mathrm{mL}, 3,91 \mathrm{ng} /$ $\mathrm{mL}, 1,95 \mathrm{ng} / \mathrm{mL}, 0,98 \mathrm{ng} / \mathrm{mL}$ e $0,49 \mathrm{ng} / \mathrm{mL}$ (Sigma, R0500, St. Louis, MO, EUA). Esses padrões foram dosados em duplicata em único ensaio.

Linearidade: seis alíquotas preparadas a partir de uma amostra com baixa concentração e outra com alta concentração por meio da mistura em diferentes proporções, e cada alíquota foi dosada em duplicata em um único ensaio.

Precisão intraensaio: 10 replicatas de um pool de amostras apresentando baixa concentração e 10 replicatas de um pool de alta concentração de 11-desoxicortisol foram dosadas em um único ensaio para determinação da precisão intraensaio.

Perfil de precisão: 88 amostras provenientes da rotina diagnóstica foram analisadas em duplicata, e o coeficiente de variação $(\mathrm{CV})$ entre as duplicadas de cada uma dessas amostras foi calculado.

Especificidade: o anticorpo empregado no ensaio, segundo dados do fabricante, apresenta reação cruzada de 5,6\% com a 17-hidroxiprogesterona (17OHP) $\mathrm{e}<1 \%$ com outros esteroides. Para investigarmos a influência dos níveis de 17OHP nas dosagens de 11-desoxicortisol por RIE, avaliamos a correlação entre o resultado desses dois hormônios em 62 amostras, nas quais foram determinadas as concentrações de $17 \mathrm{OHP}$ por RIE validado previamente pelo Laboratório de Hormônios e Genética Molecular - LIM/42 do HCFMUSP (6). Um padrão de 17OHP (Sigma, H5752, St. Louis, MO, EUA) com concentração de $1.000 \mathrm{ng} / \mathrm{mL}$ foi dosado em duplicata no RIE de 11-desoxicortisol. 
Comparação do RIE com cromatografia líquida acoplada à espectrometria de massa em tandem (LC -MS/MS): para comparação das duas metodologias, 88 amostras provenientes da rotina diagnóstica foram encaminhadas ao Laboratório Fleury para determinação de 11-desoxicortisol por LC-MS/MS descrito previamente na literatura (5).

Análise estatística: para análise estatística, foram utilizados o Programa SigmaStat 3.5 (Statcon) e o Programa EP Evaluator 9 (Data Innovations).

\section{RESULTADOS}

\section{Validação do radioimunoensaio iodado}

A sensibilidade analítica do ensaio foi de $0,30 \mathrm{ng} / \mathrm{mL}$, valor este acima do informado pelo fabricante $(0,11$ $\mathrm{ng} / \mathrm{mL})$. O estudo de exatidão com padrões Sigma em diversas concentrações evidenciou uma recuperação média de $71 \%$ (variação de $51 \%$ a $112 \%$ ), indicando que o RIE subestima os resultados de 11-desoxicortisol na ausência de outros esteroides. A linearidade mostrou-se inadequada, variando entre $75 \%$ e $102 \%$. A recuperação da alíquota de 11 -desoxicortisol foi de $75 \%$, abaixo dos valores considerados adequados ( $80 \%$ a $120 \%)$.

$\mathrm{O}$ estudo de precisão intraensaio mostrou mais uma inadequação do kit. A análise de 10 replicatas das amostras de $4,7 \mathrm{ng} / \mathrm{mL}$ e $55 \mathrm{ng} / \mathrm{mL}$ apresentou CV de respectivamente $36 \%$ e $15 \%$, valores estes acima do CV máximo permitido $(10,7 \%)$ disponível na tabela de variação biológica. No perfil de precisão, observamos que $34 \%$ das amostras apresentaram CV $\geq 20 \%$ (Figura 1). A correla-

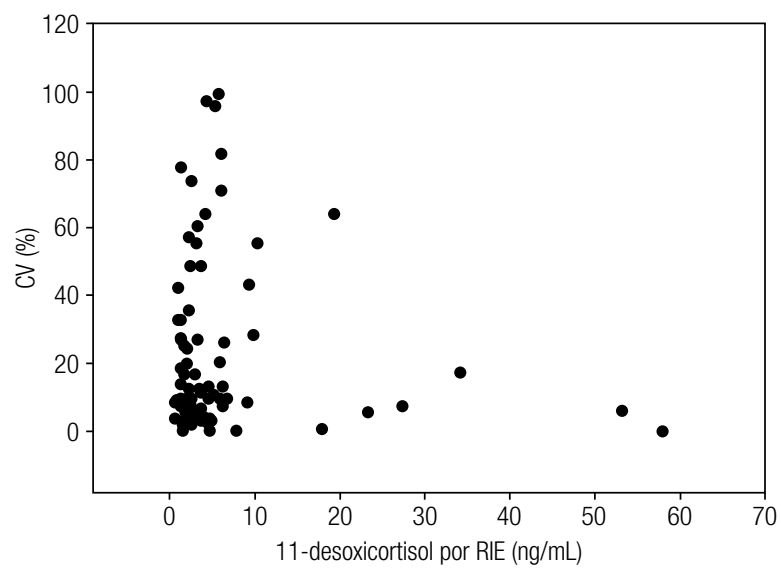

Figura 1. Avaliação do perfil de precisão. Relação entre o coeficiente de variação (CV) intraensaio e o nível médio de 11-desoxicortisol de cada amostra determinado por RIE. 0 método apresenta um perfil de precisão inadequado, sendo que mais de $30 \%$ das amostras apresentam CV maior ou igual a $10,7 \%$. ção entre o RIE e LC-MS/MS por meio da regressão de Deming resultou em um coeficiente de correlação de 0,610 , slope de 3,751, intercept de 0,145 , evidenciando uma correlação fraca entre os resultados (Figura 2). Os resultados do RIE foram sistematicamente mais altos que os observados pela técnica de LC-MS/MS, com um bias médio de 5,36 (117\%), segundo regressão de Deming.
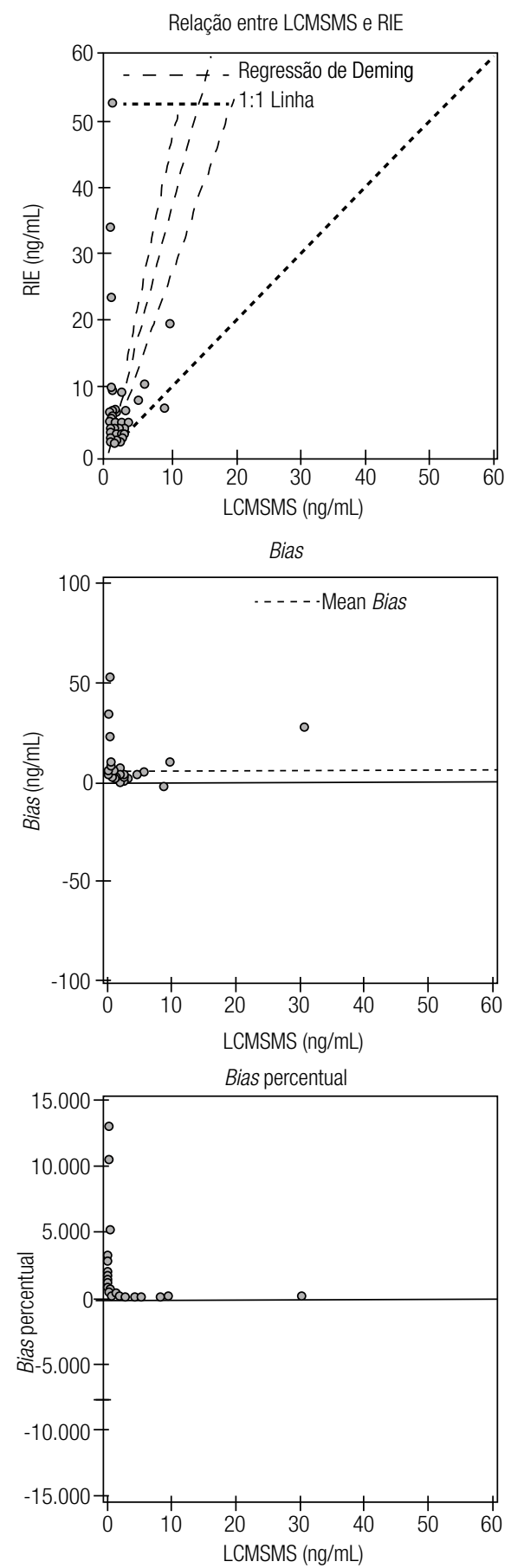

Figura 2. Comparação entre os resultados de 11-desoxicortisol obtidos por RIE e por MS/MS. Representação da relação entre os níveis de 11-desoxicortisol dosados por RIE e por LC-MS/MS ( $\mathrm{ng} / \mathrm{mL})$, bias e bias percentual utilizando LCMS/MS como referência. 


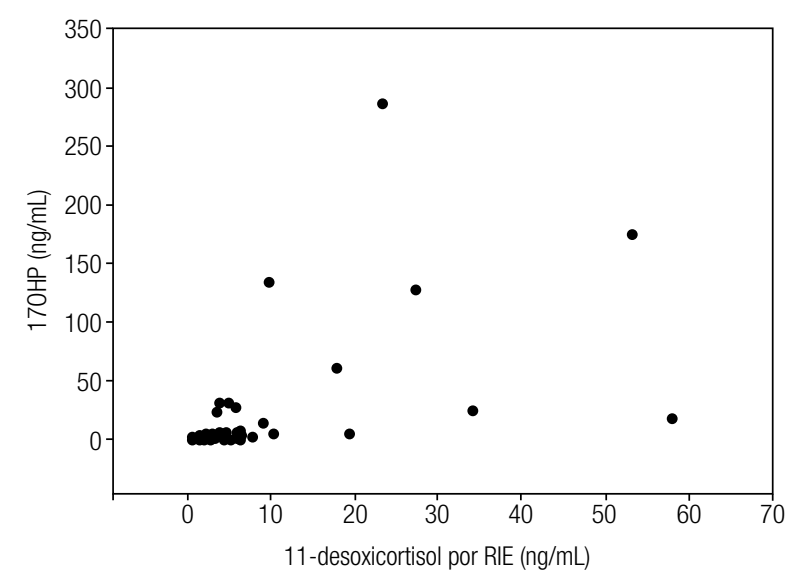

Figura 3. Correlação entre os níveis de 11-desoxicortisol e os níveis de $170 \mathrm{H}$-progesterona dosados por RIE. Representação da relação entre os níveis de 11-desoxicortisol e os de 17-hidroxiprogesterona (170HP), ambos expressos em $\mathrm{ng} / \mathrm{mL}$. A correlação entre esses valores foi baixa $(\rho=0,555)$, indicando que outros esteroides devem interferir na dosagem de 11-desoxicortisol por RIE.

A dosagem do padrão Sigma de $17 \mathrm{OHP}$ na concentração de $1.000 \mathrm{ng} / \mathrm{mL}$ resultou em valores de 11 desoxicortisol $>65 \mathrm{ng} / \mathrm{mL}$, demonstrando não ser $\mathrm{o}$ RIE um método específico.

Observamos uma correlação estatisticamente significante $(\rho=0,555, \mathrm{p}<0,001)$, entre os níveis de 17OHP e os de 11-desoxicortisol dosados por RIE, evidenciando a alta reação cruzada desse ensaio.

\section{DISCUSSÃO}

Neste trabalho, comparamos duas técnicas de dosagem do 11-desoxicortisol: a técnica de RIE e a LC-MS/MS. A espectrometria de massa é mais específica do que o radioimunoensaio para a dosagem de vários hormônios, sendo considerado o padrão-ouro para dosagem dos hormônios esteroides $(6,7)$.

As vantagens do método de RIE são sua maior disponibilidade e o menor custo para adquirir um contador de radiação $\gamma$ em relação a um cromatógrafo acoplado a um espectrômetro de massas. No entanto, na validação do método de radioimunoensaio para dosagem de 11-desoxicortisol, diversos parâmetros se mostraram inconsistentes, tornando o método impróprio para o uso na rotina diagnóstica.

Uma grave deficiência identificada pelos experimentos foi a falta de especificidade do RIE iodado, havendo reação cruzada significativa com a $17 \mathrm{OHP}$, provavelmente pela similaridade das estruturas químicas da 17OHP e do 11-desoxicortisol. Esse problema invia- biliza a aplicação do RIE como ferramenta diagnóstica, já que a dosagem de 11-desoxicortisol é fundamental para o diagnóstico diferencial entre a HAC-21OHD e a HAC-11OHD, e ambas as patologias apresentam níveis elevados de 17OHP. Esse fato foi relatado na literatura nacional em uma paciente portadora de HAC-21OHD, confirmado pela presença da mutação V281L em um dos alelos e uma grande conversão de seis éxons a partir de CYP21P no outro, que, além de apresentar níveis elevados de 17OHP (385 ng/mL) e 2l-desoxicortisol $(233,4 \mathrm{ng} / \mathrm{mL})$, também apresentava medidas elevadas de 11-desoxicortisol (49 ng/mL) segundo RIE, mostrando a ineficácia do método para determinação desse esteroide (8). Além disso, o anticorpo anti-11-desoxicortisol empregado nos RIEs apresenta reação cruzada com outros hormônios esteroides, devido à similaridade de estrutura química.

Em conclusão, o método de dosagem de 11-desoxicortisol por radioimunoensaio, atualmente disponível no mercado nacional, apresentou diversas falhas nos diferentes parâmetros avaliados, o que inviabiliza sua utilização como método de dosagem do 11 -desoxicortisol.

Agradecimentos: à Fundação de Amparo à Pesquisa do Estado de São Paulo (Fapesp) pela bolsa de iniciação científica, processo 2010/05188-0. Além disso, gostaríamos de ressaltar a colaboração dos funcionários do Setor de Hormônios do Laboratório de Hormônios e Genética Molecular - LIM/42 HCFMUSP.

Declaração: os autores declaram não haver conflitos de interesse científico neste estudo.

\section{REFERÊNCIAS}

1. Eberlein WR, Bongiovanni AM. J Clin Endocrinol Metab. 1955;15:1531-34.

2. Eberlein WR, Bongiovanni AM. Plasma and urinary corticosteroids in the hypertensive form of congenital adrenal hyperplasia. J Biol Chem. 1956;223(1):85-94.

3. Mello MP, Penachioni JY, do Amaral FC, de Castro M. Deficiência da 11 $\beta$-hidroxilase. Arq Bras Endocrinol Metab. 2004;48(5):713-23.

4. de Mello MP, BachegaTASS, da Costa-Santos M, Mermejo LM, de Castro M. Bases moleculares da hiperplasia adrenal congênita. Arq Bras Endocrinol Metabol. 2002;46(4):457-77.

5. Carvalho VM, Nakamura OH, Vieira JGH. Simultaneous quantitation of seven endogenous C-21 adrenal steroids by liquid chromatography tandem mass spectrometry in human serum. $\mathrm{J}$ Chromat B. 872(2008):154-61.

6. Bachega TA, Brenlha EM, Billerbeck AE, Marcondes JA, Madureira G, Arnhold IJ, et al. Variable ACTH-stimulated 17-hydroxyprogesterone values in 21-hydroxylase deficiency carriers are not related to the different CYP21 gene mutations. J Clin Endocrinol Metab. 2002;87(2):786-90. 
7. Lepage $\mathrm{R}$, Albert C. Fifty years of development in the endocrinology laboratory. Clin Biochem. 2006;39:542-57.

8. Tonetto-Fernandes $\mathrm{V}$, Lemos-Marini SHV, Kuperman H, RibeiroNeto LM, Verreschi ITN, Kater CE. Brazilian Congenital Hyperplasia Multicenter Study Group. Serum 21-Deoxycortisol, 17-Hy- droxyprogesterone, and 11-deoxycortisol in classic congenital adrenal hyperplasia: clinical and hormonal correlations and identification of patients with 11 beta-hydroxylase deficiency among a large group with alleged 21-hydroxylase deficiency. J Clin Endocrinol Metab. 2006;91(6):2179-84. 